\title{
Observations on Neuromuscular Dysfunction in the Addington Outbreak
}

\author{
By J. STRUAN ALEXANDER, M.B., B.S. (Lond.), M.R.C.S., L.R.C.P., D. Phys. Med. \\ Durban.
}

(Reprinted from the South African Medical Journal, Vol. 30, No. 4.)

$\mathbf{T}_{\mathrm{d}}^{\mathrm{H}}$ HIS memorandum will discuss the neuromuscular dysfunction which occurred in the majority of cases affected by the so-called Addington disease. As an approach to so limited a field it is desirable to consider briefly certain relevant clinical features.

Introduction. In the early months of 1955, and merging into the tail end of an epidemic of poliomyelitis, about 90 nurses at the Addington Hospital, Durban, and 50 civillan cases were admitted to hospital suffering from a polio-like disease. As the nature of the disease unfolded it was realized that cases of a similar character had occurred sporadically for some months before the major outbreak.

Nature of the Disease. A tentative designation of encephalomyelitis is convenient. As in poliomyelitis, onset was abrupt, with constitutional symptoms and motor paresis. But at the same time, pain was a prominent symptom, usually peripheral and in many cases protracted and severe. Moreover, the paresis did not conform to a lower-motor-neuron lesion.

Clinical Features. Constitutional symptoms included headache, prostration, malaise and mild pyrexia. Mild emotional disorder was usually expressed as euphoria, but mental depression was also noted, particularly in the later chronic stages, in which period ready fatigue and inability to concentrate were also evident. Motor involvement resulted in paresis of one or more limbs. Pain was liable to have a distribution similar to the coexistent paresis. A cardinal and confusing factor has been the variable nature of the disease, both in terms of severity and also in terms of the distribution of lesions in the selective focal points of the central nervous system. Thus, either constitutional symptoms, or motor symptoms, or sensory symptoms might predominate, and conversely one or more of these components might be minimal or absent.

It is clear therefore that the major presenting signs and symptoms are subject to considerable variation.

Course of the Disease. Onset has for the most part been abrupt. On the clinical evidence it would appear that patients progress through 3 fairly clearly defined stages, viz. the acute or active stage, the convalescent stage, and the chronic stage. It must be emphasised that the duration of these phases is variable, and has been closely related to the severity of the symptoms. In mild cases symptoms have completely cleared up after a week or two; but in many patients symptoms have smouldered for as long as 3 months, and true relapses have occurred as late as 10 weeks after onset. After subsidence of the active phase, there has been rapid and at times dramatic improvement Today, some 4 months since the first cases were notified, a residual core of patients remains hospitalized, or physically handicapped, and the long-term prognosis cannot at this stage be firmly established.

Relapses. It is suggested that relapses have been of two different types. Firstly, a minor set-back of a transient nature, in which the patient rapidly returns to the prerelapse level. A similar phenomenon is known to occur during the convalescent stage of poliomyelitis and is no more than fatigue reaction. In the second type of relapse there is evidence of renewed activity of the causative pathological process. We have seen, for example, severe pain, complete paralysis and cold sweating skin in limbs which had almost returned to normal. Hitherto unaffected limbs have also become affected. In this type of relapse recovery has followed the tardy and erratic course of an initial attack.

It is natural that many factors have been incriminated as causative of these severe relapses, but in some instances they occurred in patients under conditions of strict bed-rest.

Hysteria. As there have been overt and covert references to hysteria, this subject merits brief discussion.

Mass hysteria is surely an untenable hypothesis in view of the consistent pattern of signs and symptoms occuring in cases coming from widely scattered points in the Durban area. Encephalitic symptoms such as euphoria, mental depression, fatiguability, etc. have been a prominent feature, the locomotor disability is of a complex nature and paresis is related to volitional initiation of movement. These considerations complicate a satisfactory assessment of hysteria. In many cases a psychogenic overlay could not be ruled out.

Neuromuscular Dysfunction.

Some degree of muscle weakness has been present in the majority of cases, one or more limbs being involved, and the legs being affected more often than the arms. In a minority of cases, paresis has occurred in muscles of the back, abdomen and neck. Distribution has been asymmetrical. At onset paresis was severe and widespread, tending to involve all the joints in a limb rather than selected muscle-groups. In a few patients spasticity was present. With the recession of the acute active stage recovery of movement has been rapid for a few days. In mild paresis the improvement might be dramatic and complete, but in the more severely paretic this recession of symptoms has come to a halt at a point short of full recovery, and beyond this level progress has been tardy and subject to relapses.

When the initial diffuse weakness has receded in the lower limb, paresis has remained mainly in the hip flexors, knee extensors, and ankle dorsiflexors. In the wards the unsupported foot in a position of equinovarus was familiar, and foot-drop has been a practical difficulty in many ambulant cases. The particular pattern of movement has been sufficiently constant to form a useful guide to diagnosis. For example, with the patient lying supine and trying to raise the straight leg, there is a negative phase in which no movement takes place. As hip flexion gets going it does so in jerky fashion, with a course tremor of the whole limb. When the maximum range of hip flexion has been established, it is found that the power of the muscle is good and is able to resist counter-pressure. However, if resistance is applied at the outset, a light finger pressure is sufficient to prevent active movement.

Reflexes have for the most part shown little change; possibly they have been slightly depressed, and in patients showing spasticity clearly increased knee-jerks have been observed and have persisted. Wasting has been conspicuously minimal even after prolonged bed rest.

Electro-Diagnosis. Full investigations by current methods of strength duration curves and accommodation ratios were done on over 20 of the nurses. A smaller group 
of civilian cases were also investigated during the chronic established phase. These investigations failed to show any significant deviation from normal and may be taken to exclude destructive denervation of the lower motor neuron. Illustrative Cases.

Except for case No. 7 and 8 all the patients cited are young nurses.

Case 1. (G). First seen 2 months after onset, at which time was making good recovery from original paresis of left arm and left leg. Relapse occurred with abrupt onset, weakness of left arm only, with cold sweating of skin of hand, severe pain in shoulder and inability to move more than a flicker of fingers. Recovery of left arm has been slow and marked paresis remained 4 weeks .later.

Case 2. (F). Ten weeks after onset slight paresis of left arm, gross paresis of left leg, no active movement against gravity, coldness of left foot. Three months after onset fitted with long caliper and getting about with aid of stick.

Case 3. (B). At 10 weeks after onset, when slowly recovering from paresis of left arm and both legs and intractable headaches, suffered relapse. Right arm affected for first time. Moderate paresis but no skin coldness. At 12 weeks still a bed patient because of constitutional symptoms.

Case 4. (W). First seen 9 weeks after onset, mild paresis of left arm, both legs splinted, complaining of cramps in legs, only a flicker of active movement in toes. Mild constitutional symptoms. Responded well to active assisted movement, and within 3 weeks was ambulant and able to leave hospital.

Case 5. (M). Seen 6 weeks after onset, original paresis of right arm and right leg, later onset of paresis in both legs. At this stage marked paresis of left leg with cold foot and foot-drop; was fitted with short iron and discharged at about 11 th week.

Case 6. (H). Eleven weeks after onset showed constitutional symptoms, inability to concentrate, mild paresis in both legs, day-by-day fluctuation. When asked to raise right leg against resistance developed gross tremor which spread to left leg. Motor symptoms were subordinated to emotional state.

Case 7. (F). Onset with mild malaise (did not then or afterwards stop work), pain and paresis of left leg, pain at first simulated sciatica. Slow improvement of paresis over period of 3 months. Eight weeks after onset developed pain in left arm.

Case 8. (Mrs. B.). Severe constitutional and motor involvement. Now, 10 weeks since onset, paresis of both legs, more marked in the left. External rotation of left hip and correction is painful. Is now ambulant.

With the sudden impact of large numbers of cases of an unidentified disease, perplexing problems of diagnosis and management arose. Early anticipation of a clear-cut sequence of acute, convalescent and chronic stages have not been fulfilled. Indeed, the only constant feature has been the erratic course. With regard to morbid processes in the central nervous system, a clearer concept of their distribution and duration is fundamental to diagnosis and rational treatment.

In the early stages complete mental and physical rest are of first importance and experience has fully endorsed this principle. With the recession of symptoms we have been faced with the problem of how best to achieve the maximum recovery in disabled limbs in the shortest possible time. Essentially this amounts to the balanced application of rest and exercise, with due regard to the general condition of the patient. Appraisal of the course of paresis cannot be divorced from the concomitant emotional constitutional background.

It is now fairly well established that the answer to the therapy problem lies somewhere between the two extremes of over-zealous premature activity and unduly prolonged immobilization.

There are grounds for diversity of opinion on the degree and duration of splinting of limbs, just as is the case in the management of poliomyelitis. An objective analysis of cases treated with carefully supervised re-education of affected limbs does not indicate that this procedure has been other than helpful. Insistence on the dangers of exercise has created in the patient a resistant and apprehensive attitude that has hampered supervised reeducation of movement at a later stage. There are rational grounds for the introduction of carefully-graduated remedial exercises at an appropriate stage during recovery, with the object of facilitating volitional movement.

\section{SUMMARY.}

Neuromuscular dysfunction as it occurred in the Addington outbreak is described. Measures for physical rehabilitation are considered against the complex clinical pattern into which they had to be integrated. July 1955.

NOTE.-Although the Department of Physiotherapy at the Johannesburg General Hospital has had the opportunity of observing only one case of the Durban Mystery Disease, and therefore no conclusions can be drawn, it was interesting to read that Dr. Alexander obtained no altered electrical reactions.

In this one case the patient had a Neuroton test on three occasions, the first test two months after the initial onset of the disease, and the second one a month later, the third test being after a further month's treatment. The following results were noted:-

ACCOMMODATION TEST. (Normal is 3-6):

$$
\begin{array}{ll}
\text { December 10th, 1955 } & =2 \cdot 5 \\
\text { January 21st, 1956 } & =1.75 \\
\text { March 24th, 1956 } & =1.75
\end{array}
$$

These results are significant of a P.R.D.

\section{STRENGTH DURATION CURVE TEST.}

There were changes from the normal on each occasion in that the curve was slightly higher than normal, and irregular, which again could be interpreted as a P.R.D.

\section{CHRONAXIE.}

The muscles tested indicated a long Chronaxie.

\section{GALVANIC FARADIC TEST}

Normal i.e. the muscles showed a brisk response to both the Faradic and galvanic current, with K.C.C. greater than A.C.C.

Although the patient had voluntary movement in muscles tested, they were inclined to fatigue rapidly, and the tests showed altered responses to electrical stimuli. This I repeat cannot be conclusive as it was an isolated case, but I felt it was worth further investigation should the opportunity arise.

$$
\begin{gathered}
\text { JEAN BLAIR, } \\
\text { Senior Lecturer in Physiotherapy University of the } \\
\text { Witwatersrand. }
\end{gathered}
$$

\section{FOR SALE}

ONE ULTRA-SOUND THERAPY MACHINE-“UltraSonar", as good as new, complete with rubber-wheeled table and accessories, costing new £395. First cheque for $£ 295$ will get this machine.

Special attention is drawn to the fact that this "UltraSonar" machine has two treatment heads suitable for superficial and deep therapy. Reply:-P.O. 26, Physiotherapy Dept., General Hospital, Johannesburg. 